\title{
Antiproteinuric effect of an endothelin-1 receptor antagonist in puromycin aminonucleoside-induced nephrosis in rat
}

\author{
Jiro Kino ${ }^{1}$, Shoji Tsuji ${ }^{1}$, Tetsuya Kitao ${ }^{1}$, Yuko Akagawa ${ }^{1}$, Sohsaku Yamanouchi ${ }^{1}$, Takahisa Kimata ${ }^{1}$ and Kazunari Kaneko
}

BACKGROUND: The pathogenesis of idiopathic nephrotic syndrome (INS) remains unclear, although recent studies suggest endothelin 1 (ET-1) and CD80 of podocytes are involved. We investigated the potential of antagonist to ET-1 receptor type $A$ (ETRA) as therapeutic agent through the suppression of CD80 in a rat model of INS.

METHODS: Puromycin aminonucleoside (PAN) was injected to Wister rats to induce proteinuria: some were treated with ETRA antagonist and others were treated with 0.5\% methylcellulose. Blood and tissue samples were collected. Quantitative PCR was used to determine the expression of Toll-like receptor-3 (TLR-3), nuclear factor-kB (NF-KB), CD80, talin, ETRA, and ET-1 in the kidney. To confirm the level of CD80 protein expression, immunofluorescence staining and western blot analysis of the renal tissue were performed.

RESULTS: Amount of proteinuria in the treatment group was significantly lower than the other groups. The same-day body weight, serum creatinine values, and blood pressure were not significantly different. ETRA antagonist restores podocyte foot process effacement as well as the aberrant expression of TLR-3, nuclear factor-KB (NF-KB), and CD80 in PAN-injured kidneys.

CONCLUSIONS: The ETRA antagonist may be promising drug for INS as it showed an antiproteinuric effect. Its action was considered to be through suppression of CD80 expression on podocytes.

$\mathbf{N}$ ephrotic syndrome (NS) is characterized by heavy proteinuria. Leakage of massive amounts of serum proteins into the urine leads to a hypoalbuminemia, edema, hypercoagulable state, higher rate of infectious disease, and dysregulation of fluid balance. Childhood NS is classified into the following three types: idiopathic (idiopathic nephrotic syndrome (INS), $90 \%$ of cases), secondary (10\%), and congenital (1\%). INS is further classified into two major histological variants: minimal change NS (85\%) and focal segmental glomerulosclerosis $(10 \%)(1,2)$.

The pathogenesis of massive proteinuria in INS remains unclear, although the abnormal expression of several podocyte-related molecules such as CD80 (also known as
B7-1) or c-mip has recently attracted attention for its relationship with INS pathogenesis (3-5).

Endothelin (ET), which has a potent vasoconstrictor effect, was shown to be expressed not only in endothelium but also in podocytes, and the potential for ET to cause structural changes of podocytes leading to proteinuria has been reported $(6-8)$. Therefore, the possibility of using a therapeutic drug that is an ET receptor type A (ETRA) antagonist has gained prominence. In fact, it has been reported to reduce proteinuria and prevent glomerulosclerosis (9-11), although its mechanisms of action remain to be clarified.

Taken together, we wonder whether ETRA antagonist (ambrisentan) ameliorates proteinuria in INS through modification of podocyte-related molecules including CD80. To test this hypothesis, we (i) determined the ability of ambrisentan to reduce proteinuria in rats with puromycin aminonucleoside (PAN)-induced nephrosis, an animal model of INS, and (ii) analyzed the potential molecular mechanisms of action of ambrisentan on kidneys of rats with nephrosis induced by PAN.

\section{METHODS}

\section{Animal and Protocol}

The protocol was approved by Kansai Medical University (no.15065). Female Wister rats (6-week old) were purchased from CLEA Japan (Tokyo, Japan). PAN nephrosis was induced by a single intravenous injection of PAN (Sigma, St. Louis, MO) dissolved in phosphate-buffered saline (PBS) at a dose of $50 \mathrm{mg} / \mathrm{kg}$ body weight. Ambrisentan was purchased from Glaxo Smith Kline (Brentford, UK). Systolic blood pressure was measured using a rat manometer (Brain Science Idea, Tokyo, Japan). A group of PAN-injected rats $(n=15)$ was left untreated and received sham oral gavage vehicle solution $(0.5 \%$ methylcellulose), whereas the treatment group also received ambrisentan $(10 \mathrm{mg} / \mathrm{kg}, n=15)$ dissolved in $0.5 \%$ methylcellulose orally once daily beginning 2 days before PAN injection and until day 9 . Five control rats also received $0.5 \%$ methylcellulose by oral gavage daily with PBS injection. On day 9 or 12, the rats were anesthetized by isoflurane inhalation. The kidneys were removed immediately, and the renal cortex was processed. During the course of the experiments, 24-h urine specimens were obtained from all rats in metabolic cages each day, and urinary protein was measured using the pyrogallol red method. Serum creatinine level was measured by standard laboratory methods.

\section{Transmission Electron Microscopy}

For ultrastructural analysis, the renal cortex was dissected after systemic perfusion with PBS. Immediately, the tissues were fixed in 


\section{Articles | Kino et al.}

$2 \%$ glutaraldehyde for overnight, post-fixed in $1 \%$ osmium tetroxide, dehydrated in a graded acetone series, and embedded in EponAraldite. Ultrathin sections, cut to $0.08 \mu \mathrm{m}$ thick and stained with uranyl acetate and lead citrate, were examined using a Hitachi H-7100 (Tokyo, Japan).

RNA Extraction and Quantitative Reverse Transcription-PCR Total cellular RNA was extracted from the processed renal cortex using the RNeasy Mini Kit (QIAGEN, Hilden, Germany). To quantify mRNA expression, the real-time SYBR-Green assay was performed. For this, $1 \mu \mathrm{g}$ of RNA was converted to single-strand DNA (TAKARA, Shiga, Japan). Each cDNA was mixed with $10 \mu \mathrm{M}$ of forward and reverse primers and $12.5 \mu \mathrm{l}$ of SYBR Premix Ex Taq II (TAKARA). Quantitative PCR was performed in an Applied Thermal Cycler Dice Real Time System II (TAKARA). The following primers were used:

TLR-3 forward, 5'-AGGTATTGAACCTGCAACACAATGA-3'; TLR-3 reverse, 5'-CCCAAGTTCCCAACTTTGTAGATGA-3';

Nuclear factor- $\kappa \mathrm{B}(\mathrm{NF}-\kappa \mathrm{B})$ forward, $5^{\prime}$-GATGGGACGACACCTC TACACATA-3';

NF- $\kappa$ B reverse, 5'-CCCAAGAGTCGTCCAGGTCA-3';

CD80 forward, 5' -TCGTAGGTGAAACACCTGA-3';

CD80 reverse, 5'-CCGGAAGCAAAGCAGGTAATC-3';

Talin forward, 5'-TGTGCCAATGGCTACCTGGA-3';

Talin reverse, 5'-GTCCTGTCAGCTGCTGCTTTAGTTC-3';

Ednra forward, 5'-GCATTAACCTGGCAACCATGAAC-3';

Ednra reverse, 5'-GGACTGGTGACAACAGCAACAGA-3';
ET-1 (endothelin 1) forward, 5'-ACCTGGACATCATCTGGGT CAAC-3';

ET-1 reverse, 5'-TTTGGTGAGCACACTGGCATC-3'; GAPDH forward, 5'-GGCACAGTCAAGGCTGAGAATG-3'; GAPDH reverse, 5'-ATGGTGGTGAAGACGCCAGTA-3'.

The threshold cycle $(\mathrm{Ct})$ was used for determining the relative expression level of each gene, by normalizing to the Ct of GAPDH. The $\Delta \Delta \mathrm{CT}$ was used to calculate the relative change.

\section{Immunofluorescence Staining}

In order to confirm the presence of CD80 protein expression in the kidney, immunofluorescence staining was performed at Biopathology Institute (Oita, Japan). Kidneys were fixed in $4 \%$ paraformaldehyde. Sections were mounted on silane-coated slides in 3 - $\mu$-thick paraffin sections. After deparaffinized sections had been autoclaved at $120^{\circ} \mathrm{C}$ for $10 \mathrm{~min}$ to block endogenous peroxidase activity, they were incubated at $4{ }^{\circ} \mathrm{C}$ for $1 \mathrm{~h}$ with anti-CD80 primary antibody (OriGene, Rockville, MD) in Tris-buffered saline at 1:100 dilution and SlowFade Gold Antifade Mountant with 4',6-diamidino-2-phenylindole (DAPI) (Thermo Fisher Scientific, Waltham, MA). Sections were then incubated with fluorescent secondary antibody (Alexa Flour 488 Goat anti-mouse IgG; Invitrogen, Carlsbad, CA) in Tris-buffered saline for $1 \mathrm{~h}$ at room temperature and mounted with Prolong Gold Antifade Reagent (Invitrogen). Kidney sections were viewed and imaged with an OLYMPUS DP70 (OLYMPUS, Tokyo, Japan).

\section{Western Blot Analysis}

In order to confirm the expression of CD80 on kidney at a protein level, western blot analysis was also performed. Protein extracts from

\section{b}
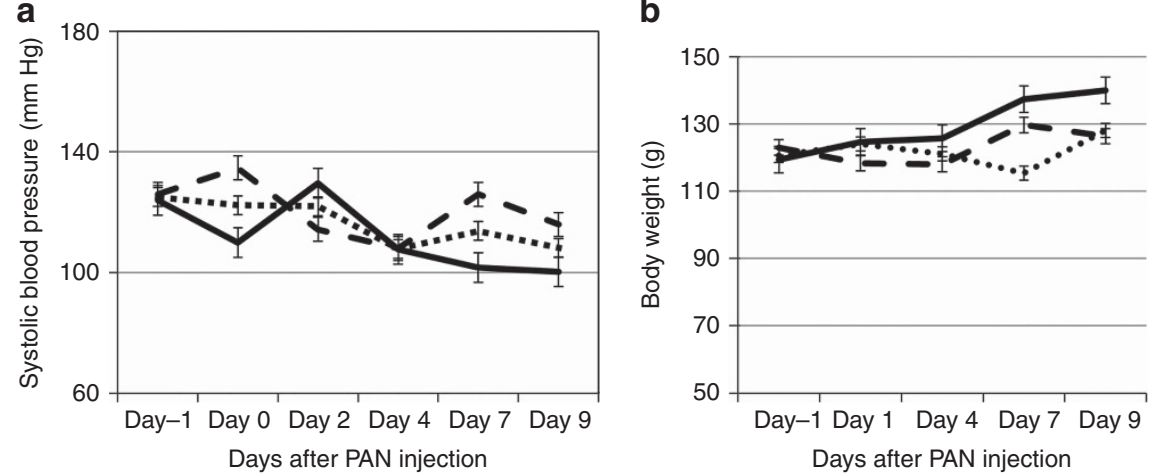

C

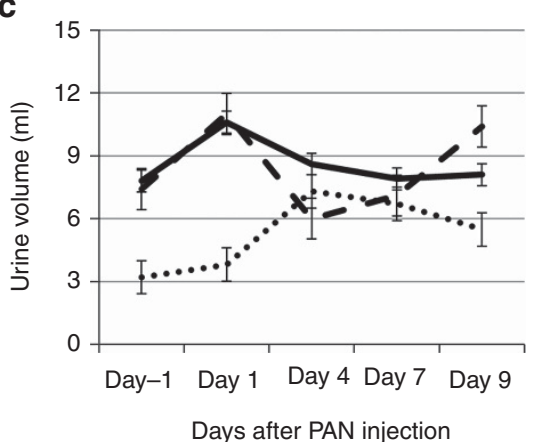

d

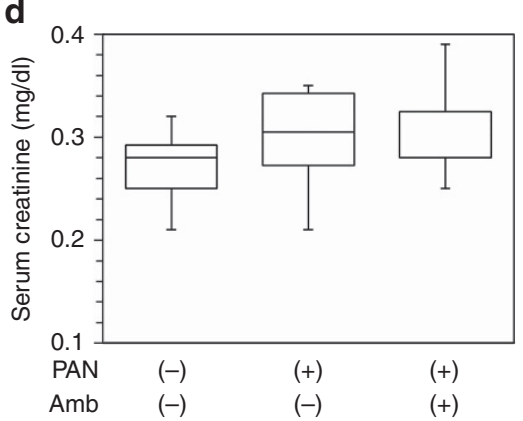

Figure 1. Evaluation of body weight, blood pressure, and urine volume. (a) There was no significant difference among the groups in terms of systolic arterial pressure (SAP) by tail cuff measurement. (b) Regularly measured body weights were not different among the three groups. (c) There was no significant change in urine volume between the PAN and the treatment group. (d) No statistically significant differences in serum creatinine values were found among the three groups of rats on day 9, either. (a-c) Solid line: rats injected with PAN and ambrisentan; broken line: rats injected with PAN; dotted line: rats received no treatment except PBS injection. All error bars in the graphs represent SEM. (d) The central horizontal line in the box represents the median value, and the bottom and top edges of the box are located at the 25th and 75th percentiles, respectively. The central vertical lines extend from the box to the 90th or 10th percentiles. PAN, Puromycin aminonucleoside; PBS, phosphate-buffered saline. 
kidneys $(40 \mu \mathrm{g})$ were separated by SDS-polyacrylamide gel electrophoresis, and transferred to poly vinylidene di-fluoride membrane (Bio-Rad, Hercules, CA). Membranes were blocked for $1 \mathrm{~h}$ with Blocking One (nacalai tesque, Kyoto, JAPAN), followed by incubation in primary antibody overnight (anti-CD80 (OriGene); anti-GAPDH (Cell Signaling Technology, Danvers, MA)). Blots were then incubated in horseradish peroxidase-conjugated secondary antibodies against mouse or rabbit IgG (Abcam, Cambridge, UK) for $1 \mathrm{~h}$ at room temperature. Protein bands were detected using the enhanced chemiluminescence (ECL) reagent (Bio-Rad). Membranes were scanned using a LAS4000 (GE Healthcare Bio-Sciences, Pittsburgh, PA).

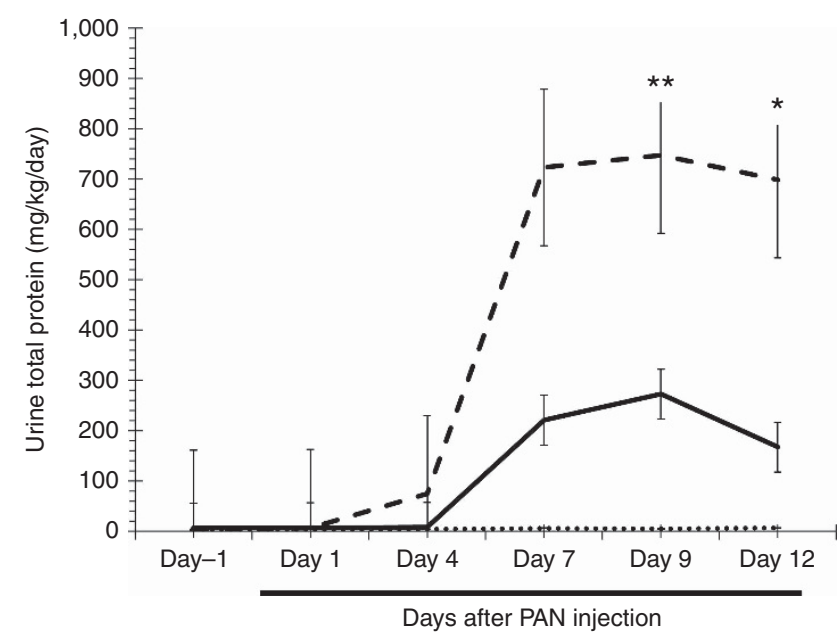

Figure 2. Detailed analyses of proteinuria in PAN-injected Wister rats. PAN injection without ambrisentan in the Wister rats $(n=10)$ induced proteinuria, which gradually increased in its amount up to day 9 and gradually decreased thereafter (broken line). In contrast, treatment group with $10 \mathrm{mg} / \mathrm{kg}$ of ambrisentan $(n=10)$ significantly reduced the amount of proteinuria during days 7,9 , and 12 (solid line). The control rats receiving intravenous PBS maintained baseline levels of urinary protein even on day 9 (dotted line). During the course of the experiments, 24-h urine specimens were obtained from all rats in metabolic cages each day, and urinary protein was measured using the pyrogallol red method. ${ }^{*} P<0.05 ;{ }^{*} P<0.01$. All error bars in the graphs represent SEM. PAN, puromycin aminonucleoside.

\section{Statistical Analysis}

Two-way ANOVA was used for statistics among three experimental groups followed by a Tukey's test for post hoc analysis. Significance was defined as $P<0.05$. All error bars in the graphs represent SEM.

\section{RESULTS}

Evaluation of Body Weight, Blood Pressure, Urine Volume, and Serum Creatinine Level

At first, we evaluated whether ambrisentan has an impact on body weight and systemic blood pressure using every three rats on day -1 , day 1 , day 4 , day 7 , and day 9 . As a result, blood pressure had no significant difference in all dates (Figure 1a). There was no significant difference in body weight in the same way (Figure 1b). Furthermore, in this experiment, there was no significant change in the urine volume and serum creatinine level between the PAN and the treatment group (Figure 1c,d).

\section{Ambrisentan Reduces PAN-Induced Proteinuria in Rats}

As shown in Figure 2, 10 female Wistar rats developed massive proteinuria after a single intravenous injection of PAN $(50 \mathrm{mg} / \mathrm{kg}$ ), which peaked on day 9 (urinary total protein [U-TP] $747.7 \mathrm{mg} / \mathrm{kg} /$ day: dashed line). In contrast, five control rats receiving intravenous PBS maintained baseline levels of urinary protein (U-TP on day 9: $4.7 \mathrm{mg}$ / $\mathrm{kg}$ /day: dotted line). To determine the ability of ambrisentan to reduce PAN-induced proteinuria, 10 rats/group were also treated daily with oral administration of ambrisentan $(10 \mathrm{mg} /$ $\mathrm{kg}$ ), which significantly reduced proteinuria (U-TP on day 9: $272.6 \mathrm{mg} / \mathrm{kg} /$ day: solid line). Thus, ambrisentan treatment prevented PAN-induced proteinuria.

\section{Ambrisentan Restores Foot Process Effacement in PAN-Injured Glomeruli}

Evaluation of glomeruli by electron microscopy revealed that PAN-injured glomeruli showed structural changes of podocytes (Figure 3a), with their edema and foot process
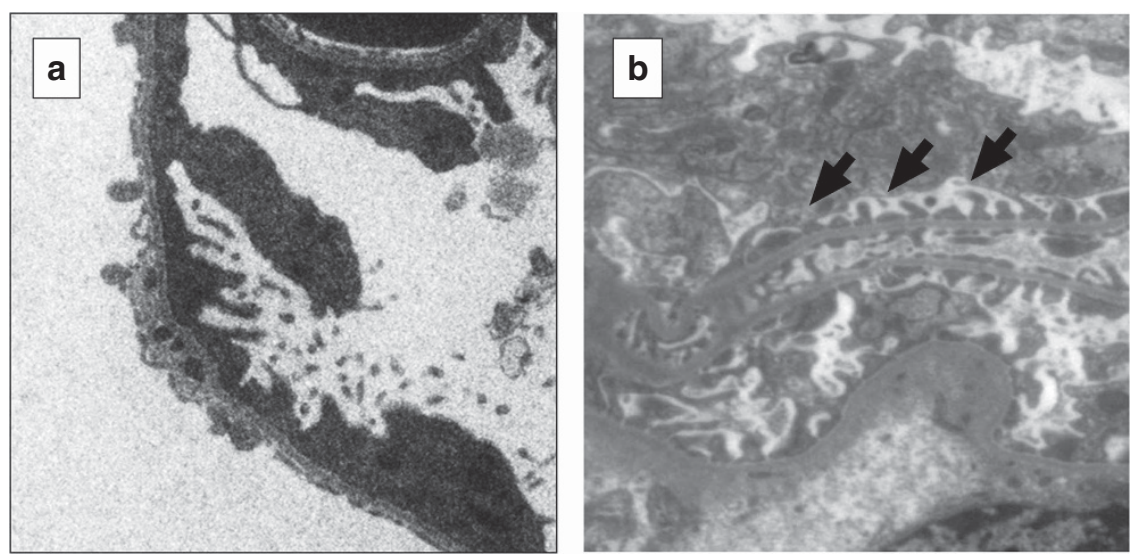

Figure 3. Electron microscopy analyses of the kidneys of PAN-injected rat with or without ambrisentan. The panels show representative images of the electron microscopy in the glomeruli of kidneys, 9 days after PAN injection. Electron microscopic analysis revealed podocyte flattening and extensive effacement of foot processes only in the glomeruli of the PAN-injected rat $(\mathbf{a}, \times 2,000)$ but not in the glomeruli of the PAN-injected rat pretreated with ambrisentan (arrows in $\mathbf{b}, \times 1,800$ ). PAN, puromycin aminonucleoside. 
a

a

4

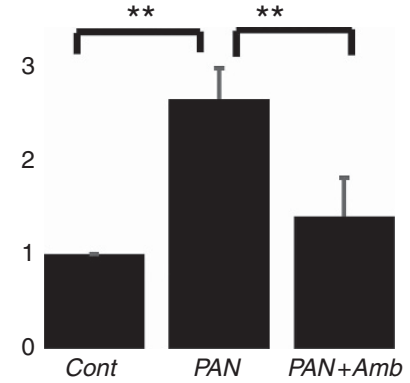

$\mathbf{d}$
1.5

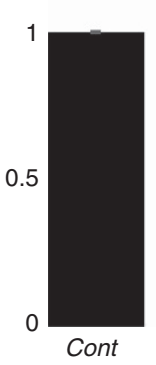

TLR-3

Talin

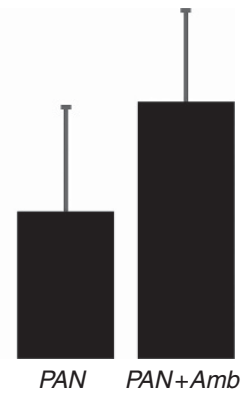

e

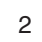

1.5 b

1.5

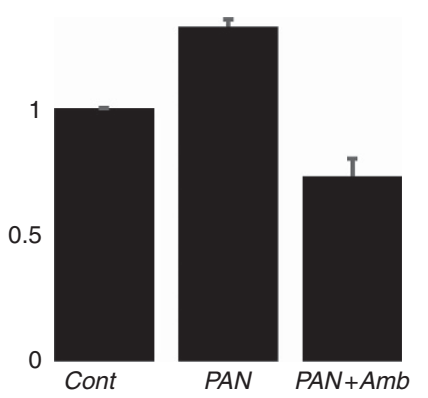

$N F-\kappa B$
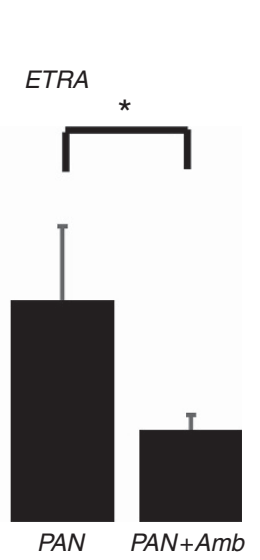

c

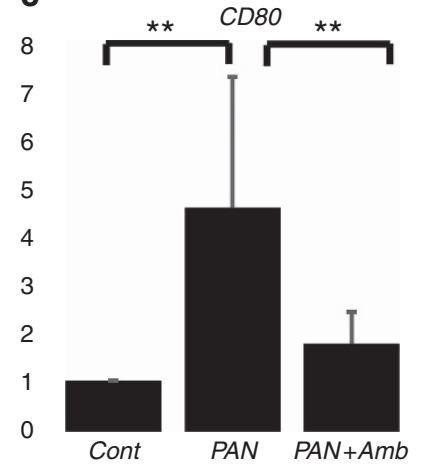

f

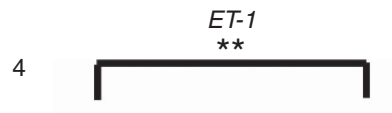

3
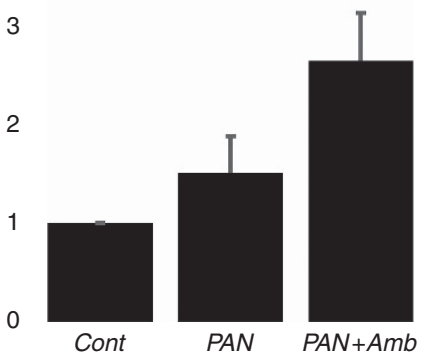

Figure 4. Ambrisentan restores both the enhanced expression of TLR-3 and CD80 in kidney tissues of PAN-induced injury. Total RNA was extracted from dissected renal cortex from control (Cont), PAN-injected (PAN), and PAN-injected rats treated with ambrisentan (PAN + Amb; $n=5$ per each groups). (a-c) Glomerular mRNA expression of TLR-3 (encodes for TLR-3) and CD80 (encodes for CD80) was significantly increased after PAN-induced injury. These enhanced expressions were significantly inhibited by treatment with ambrisentan. Glomerular mRNA expression of NF-KB (encodes for NF-KB) indicated a similar tendency, although it did not reach statistical significance. (d) In contrast, glomerular mRNA expression of talin was suppressed by PAN injection, which was restored by ambrisentan, although it did not reach statistical significance either. (e,f) Regarding glomerular mRNA expression of ETRA (encodes for ETRA) and ET-1 (encodes for ET-1), they were not significantly affected by PAN injection, whereas treatment with ambrisentan decreased glomerular mRNA expression of ETRA and increased glomerular mRNA expression of $E T-1$. ${ }^{*} P<0.05$, ${ }^{* *} P<0.01$. All error bars in the graphs represent SEM. ET-1, endothelin 1; ETRA, ET-1 receptor type A; NF-KB, nuclear factor-kB; PAN, puromycin aminonucleoside; TLR, Toll-like receptor-3.

effacement. In contrast, ambrisentan treatment after PAN injection prevented the severity of foot process effacement compared with PAN injection alone (Figure $3 \mathbf{b}$ ).

\section{Ambrisentan Restores both the Expression of mRNA of TLR-3,} NF-KB, CD80, and talin in Kidney Tissues

Enhanced glomerular mRNA expression of TLR-3 (encodes for Toll-like receptor-3 (TLR-3)), NF- $\kappa B$ (encodes for NF- $\kappa B$ ), and $C D 80$ (encodes for CD80) was inhibited after treatment with ambrisentan (Figure 4a-c). In contrast, glomerular mRNA expression of talin was suppressed by PAN injection, which was restored by ambrisentan (Figure 4d). Regarding glomerular mRNA expression of ETRA (encodes for ETRA) and ET-1 (encodes for ET-1), they were not significantly affected by PAN injury. However, treatment with ambrisentan decreased glomerular mRNA expression of ETRA and increased that of $E T-1$ (Figure $4 \mathrm{e}, \mathrm{f}$ ). ${ }^{\star} P<0.05$. All error bars in the graphs represent SEM.

\section{Ambrisentan Restores CD80 Expression in PAN-Injured Glomeruli}

Immunofluorescence staining of CD80 in glomeruli and western blot analysis using protein extracts from kidneys showed a significant increase in CD80 expression in PANinduced injury, which was restored after the treatment with ambrisentan (Figure 5a,b). Thus, these results indicate an association between CD80 expression and urinary protein loss.

\section{DISCUSSION}

ET, which was discovered in 1988 , is a powerful 21 -aminoacid vasoconstrictive peptide that originates in the endothelium. There are three types of the ET peptide family (ET-1, 2, and 3), which are found in most mammals including humans; among them, ET-1 shows the strongest physiological effects $(12,13)$. ET-1 production in endothelial cells is promoted by bioactive substances, including transforming growth factor- $\beta$, vasopressin, adrenalin, and other cytokines, whereas its 


\section{Antiproteinuric effect of ambrisentan Articles}
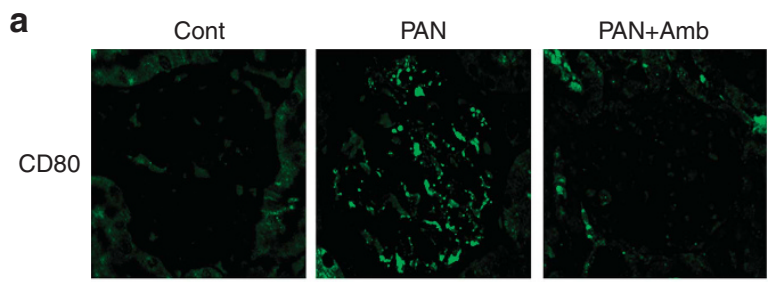

b
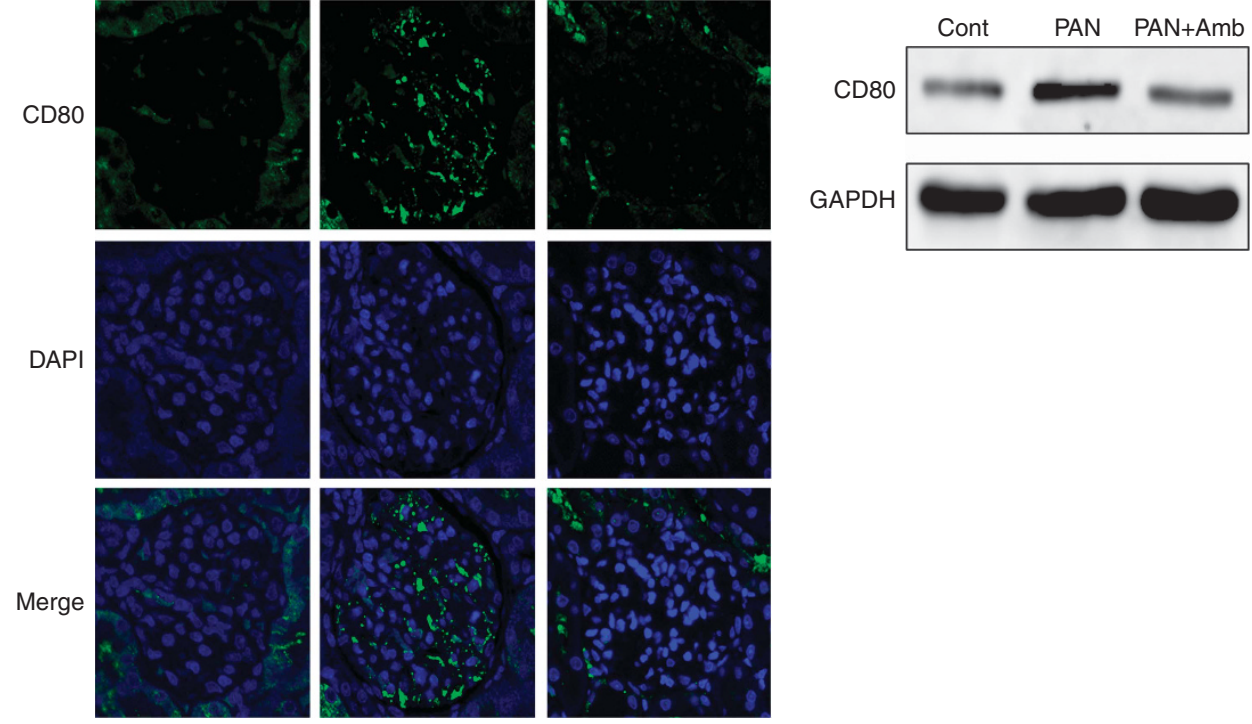

Figure 5. Ambrisentan restores the enhanced protein expression of CD80 in kidney tissues of PAN-induced injury. (a) The panels show representative images of the immunofluorescence analysis of glomeruli from control (Cont), PAN-injected (PAN), and PAN-injected rats treated with ambrisentan (PAN+Amb). Ambrisentan restored the enhanced CD80 expression in PAN-injected glomeruli of rats. Green fluorescence denotes CD80 in the glomeruli and blue fluorescence (DAPI) is indicative of the presence of a nucleus. Original magnification $\times 300$. (b) Representative images of western blot analysis showing the restoration of normal CD80 expression, which was enhanced as a result of PAN injection in rats treated with ambrisentan. PAN, puromycin aminonucleoside.

production is inhibited by nitric oxide, prostacyclin, atrial natriuretic peptide, and others $(14,15)$. ET-1 combines with two receptors of type A and type B (ETRB). When these receptors are stimulated by ET-1, various intracellular signaling systems such as the protein kinase C pathway or mitogen-activated protein kinase activation pathway are activated $(16,17)$. ET-1-mediated activation of these receptors is known to be involved in pulmonary hypertension and hypertensive status $(15,18)$. Therefore, various ET receptor antagonists, including selective ETRA antagonist and ETRB antagonist, as promising agents for pulmonary hypertension are currently under development $(19,20)$.

ET family peptides are found in endothelial cells as well as in all other organs, including the kidneys (21): ET production occurs in the glomerular endothelial cells, podocytes, or renal tubular cells $(22,23)$. Excessive or decreased production of ET-1 is thought to cause disequilibrium between the two types of receptors, leading to proteinuria and kidney diseases $(6,8)$. It has been postulated that numerous ET-dependent mechanisms contribute to proteinuria and kidney diseases $(24,25)$ : ET promotes collagen production and stimulates glomerular fibronectin synthesis; ET becomes activated under conditions associated with renal disease progression, such as diabetes, insulin resistance, obesity, dyslipidemia, reactive oxygen species formation, and inflammation $(26,27)$.

An involvement of ET-1 in the development of proteinuria in INS was suggested by an increase of ET-1 excreted into the urine of the patients (28). An animal study using PANinduced nephrosis in rats further supported an important role of ET-1 in INS as it demonstrated a significant decrease in proteinuria following treatment with an ETRA antagonist, although the contradicting results also existed $(29,30)$. However, to the best of our knowledge, there have been no studies to investigate the molecular mechanisms within podocytes in the development of ET-1-induced proteinuria.

Regarding the mechanisms of proteinuria in INS, it was recently hypothesized that structural changes occur because of immunological abnormalities that activate podocytes (two-hit theory) (31). This theory is illustrated in Figure 6 and is summarized as follows: (i) some types of antigens (viral RNA or serum protein) bind to the TLRs present on the podocytes (first hit); (ii) NF- $\mathrm{BB}$ of podocytes is activated by TLR stimulation; (iii) NF- $\mathrm{KB}$ activation causes overexpression of CD80 on podocytes leading to suppression of $\beta 1$ integrin by blocking the binding of talin to the $\beta 1$ integrin; (v) because of these structural changes, serum proteins from glomerular capillaries leak into the urine; and (vi) regulatory $\mathrm{T}$ cells that control the expression of CD80 circulating in the blood are functionally or quantitatively reduced (second hit). Continued proteinuria sustains the abnormal condition described above (i-vi) (32). In this theory, central factor is CD80, which is a $53-\mathrm{kD}$ membrane-associated protein. It had been long believed that CD80 was only expressed on B lymphocytes and antigen-presenting cells where it acts as a co-stimulatory receptor with role in T-cell activation $(33,34)$. The role of CD80 in podocyte pathobiology was proposed by Reiser $e t$ al. in an experimental model where lipopolycaccharide injection in mice resulted in increased expression of CD80 in podocytes and proteinuria, whereas no proteinuria occurred in CD80 knockout mice (3). Furthermore, Yu et al. have recently found 


\section{Articles $\mid$ Kino et al.}

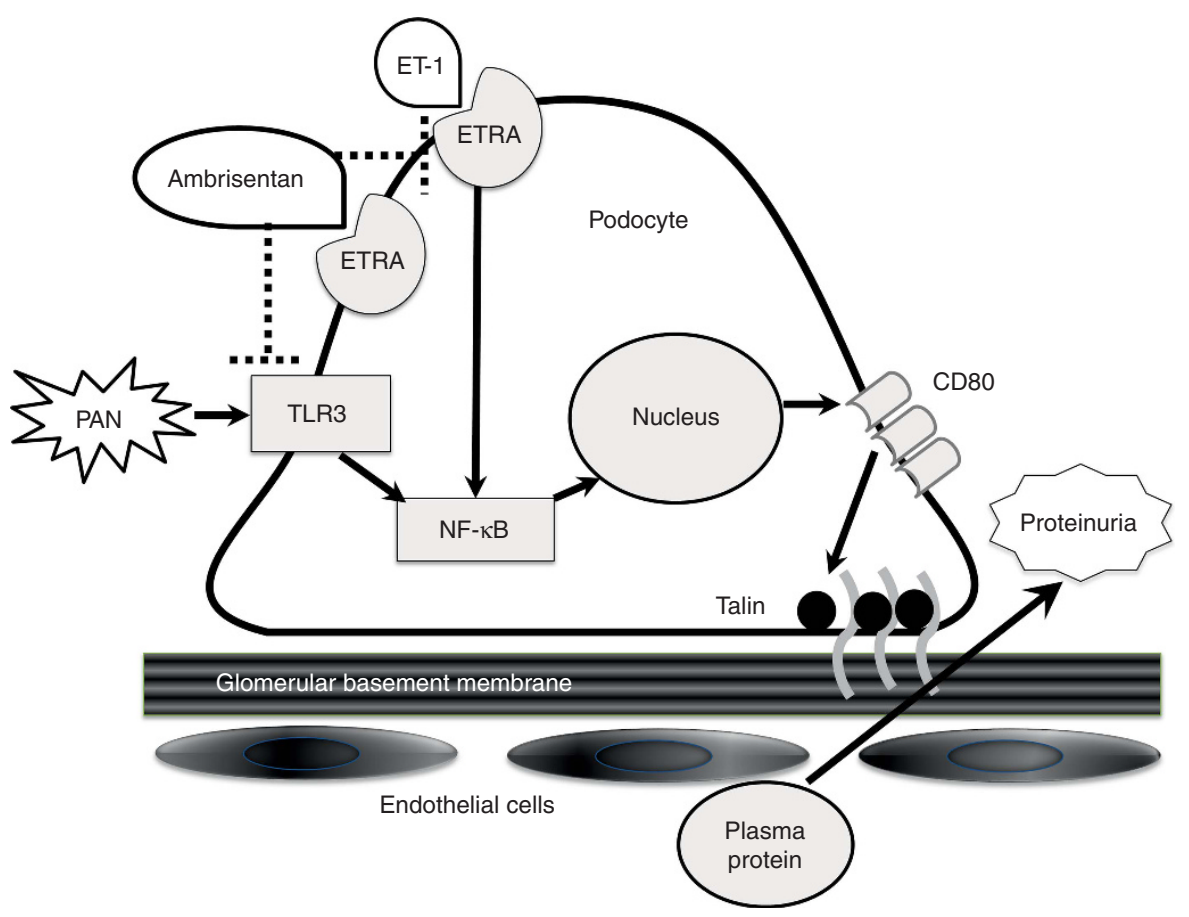

Figure 6. Hypothetical molecular mechanism of podocyte on the appearance of proteinuria in PAN-injected rats. Stimulation by PAN administration caused activation of the NF-KB intracellular signaling pathway within podocytes through TLR-3. Activated podocytes express CD80 on cellular surfaces. Overexpressed CD80 downregulated talin expression resulting in detachment of podocyte from the glomerular basement membrane. Upon administration of the ambrisentan, this signaling pathway was suppressed by downregulating the expression of TLR-3, NF-KB, and CD80 by several possible cascades, and these restored the talin expression and reduced the amount of proteinuria. Solid arrow lines indicate the stimulation or activation, whereas dashed lines denote the inhibitory signals. NF-KB, nuclear factor-kB; PAN, puromycin aminonucleoside; TLR, Toll-like receptor-3.

that CD80-positive podocytes have a reduced capacity to attach to the surrounding matrix through $\beta 1$ integrin. They found that CD80-positive podocytes change their morphologic characteristics, leading to podocyte foot processes' detachment from the glomerular basement membrane and thus proteinuria (35). Recent report that children with steroid-sensitive NS are characterized by increased expressions of TLR-3, TLR-4, and CD80 mRNA in peripheral blood mononuclear cells also lends support for this theory (36).

In this study, we evaluated this theory using an in vivo animal model of INS to clarify the molecular pathway within podocytes in ET-1-induced proteinuria and therapeutic potential of the selective ETRA antagonist such as ambrisentan. We used PAN as a nephrotoxin because it is commonly used to induce massive proteinuria in rats. In addition, it has an advantage compared with the other nephrotoxins for the study of the pathogenesis of proteinuria in INS because rats develop massive proteinuria within 10 days after its single injection and is known to induce foot process effacement, a hallmark of INS. Furthermore, the previous reports that PAN-induced CD80 activation in cultured podocytes (3) prompted us to choose it. As a result, an administration of ambrisentan to PAN rats showed effects against proteinuria without significant changes in serum creatinine, urine volume, and blood pressure. The suppression of effacement of podocyte foot process was also confirmed using electron microscopy. Study with reverse transcriptase
PCR (RT-PCR) using the renal tissue from PAN-induced nephrosis rats treated with ambrisentan disclosed the following characteristics: (i) suppressed mRNA expression of TLR-3, NF- $\mathrm{kB}$, and CD80, all of which were upregulated by PAN-induced nephrosis; (ii) increased mRNA expression of talin-1, which has an important role of attachment of podocytes to the surrounding matrix through $\beta 1$ integrin; (iii) although glomerular mRNA expression of ETRA and ET-1 were not significantly affected by PAN injury, treatment with ambrisentan decreased glomerular mRNA expression of ETRA and increased that of ET-1 in agreement with the previous report (37). These findings suggest the existence of feedback loops on production of ETRA and ET-1 within renal glomeruli (6). Furthermore, we confirmed the CD80 protein expression in the PAN-induced nephritic kidney by immunohistochemical and western blot analysis.

On the basis of these results, the following can be predicted regarding the appearance of proteinuria in PAN-injected rats and the antiproteinuric effects of ambrisentan (Figure 6): stimulation by PAN administration caused activation of the NF- $\kappa B$ intracellular signaling pathway within podocytes through TLR-3; activated podocytes express CD80 on cellular surfaces; overexpressed CD80 downregulated talin expression resulting in detachment of podocyte from the glomerular basement membrane (38); upon administration of the ambrisentan, this signaling pathway was suppressed by downregulating the expression of TLR-3, NF- $\mathrm{KB}$, and CD80 
by several possible cascades and these restored the talin expression and reduced the amount of proteinuria.

A limitation of this experiment was that we could examine the expression of only six molecules in the podocytes. Some reports have suggested that ET-1 stimulation via ETRA induces signal transmission by mitogen-activated protein kinase, matrix metalloprotease-9, Rho-kinase, and other pathways, causing changes to the podocyte cytoskeleton and structural changes to the slit membranes $(10,26,39)$. These signaling pathways might be also involved. In addition, as we have not performed the experiments using CD80 knockout mice, we cannot conclude whether CD80 is the absolute cause of proteinuria.

In conclusion, our results suggest that ambrisentan acts on podocytes to exhibit an antiproteinuric effect that is through the NF- $\mathrm{kB}$ intracellular signaling pathway and is less likely to act through renal hemodynamics in PAN-induced nephrosis in rats, an animal model of INS. Therefore, ETRA antagonist such as ambrisentan may have potential as a therapeutic agent for INS.

\section{ACKNOWLEDGMENTS}

We thank A. Yoshioka at Kansai Medical University for their expert technical assistance, and also T. Katsuta in the Department of Pediatrics, Kansai Medical University, for aid in the preparation of this manuscript. We also would like to thank Shipra Agrawal for reading of the manuscript, Editage (www.editage.jp) for English language editing. Part of this work was presented as a poster at the American Society of Nephrology (ASN) Kidney Week, 16-19 November 2016, Chicago, IL.

\section{STATEMENT OF FINANCIAL SUPPORT}

This work was supported by Grants-in-Aid for Scientific Research (C) from the Japan Society for the Promotion of Science (KAKENHI, Grant numbers 25461634).

Disclosure: The authors declare no competing financial interests.

\section{REFERENCES}

1. Eddy AA, Symons JM. Nephrotic syndrome in childhood. Lancet 2003;362:629-39.

2. KDIGO. Chapter 3: steroid-sensitive nephrotic syndrome in children. Kidney Int Suppl 2012;2:163-71.

3. Reiser J, von Gersdorff G, Loos M, et al. Induction of B7-1 in podocytes is associated with nephrotic syndrome. J Clin Invest 2004;113:1390-7.

4. Audard V, Zhang SY, Copie-Bergman C, et al. Occurrence of minimal change nephrotic syndrome in classical Hodgkin lymphoma is closely related to the induction of c-mip in Hodgkin-Reed Sternberg cells and podocytes. Blood 2010;115:3756-62.

5. Cara-Fuentes G, Clapp WL, Johnson RJ, Garin EH. Pathogenesis of proteinuria in idiopathic minimal change disease: molecular mechanisms. Pediatr Nephrol 2016;31:2179-89.

6. Barton M, Tharaux PL. Endothelin and the podocyte. Clin Kidney J 2012;5:17-27.

7. Dhaun N, Webb DJ, Kluth DC. Endothelin-1 and the kidney-beyond BP. Br J Pharmacol 2012;167:720-31.

8. Lenoir O, Milon M, Virsolvy A, et al. Direct action of Endothelin-1 on podocytes promotes diabetic glomerulosclerosis. J Am Soc Nephrol 2014;25:1050-62.

9. Ebihara I, Nakamura T, Tomino Y, Koide H. Effect of a specific endothelin receptor A antagonist and an angiotensin converting enzyme inhibitor on glomerular mRNA levels for extracellular matrix components, metalloproteinases (MMP) and a tissue inhibitor of MMP in aminonucleoside nephrosis. Nephrol Dial Transplant 1997;12:1001-6.

10. Ortmann J, Amann K, Brandes RP, et al. Role of podocytes for reversal of glomerulosclerosis and proteinuria in the aging kidney after endothelin inhibition. Hypertension 2004;44:974-81.

11. Wenzel RR, Littke T, Kuranoff S, et al. Avosentan reduces albumin excretion in diabetics with macroalbuminuria. J Am Soc Nephrol 2009;20:655-4.

12. Yanagisawa $M$, Kurihara $H$, Kimura $S$, et al. A novel potent vasoconstrictor peptide produced by vascular endothelial cells. Nature 1988;332:411-5.

13. Furchgott RF, Vanhoutte PM. Endothelium-derived relaxing and contracting factors. FASEB J 1989;3:2007-18.

14. Terada Y, Tomita K, Nonoguchi H, Marumo F. Different localization of two types of endothelin receptor mRNA in microdissected rat nephron segments using reverse transcription and polymerase chain reaction assay. J Clin Invest 1992;90:107-2.

15. Kohan DE, Rossi NF, Inscho EW, Pollock DM. Regulation of blood pressure and salt homeostasis by endothelin. Physiol Rev 2011;91:1-77.

16. Kohan DE, Inscho EW, Wesson D, Pollock DM. Physiology of endothelin and the kidney. Compr Physiol 2011;2:883-919.

17. De Miguel C, Speed JS, Kasztan M, Gohar EY, Pollock DM. Endothelin-1 and the kidney: new perspectives and recent findings. Curr Opin Nephrol Hypertens 2016;25:35-41.

18. Sauvageau S, Thorin E, Caron A, Dupuis J. Evaluation of endothelin-1induced pulmonary vasoconstriction following myocardial infarction. Exp Biol Med 2006;231:840-6.

19. Macchia A, Marchioli R, Tognoni G, et al. Systematic review of trials using vasodilators in pulmonary arterial hypertension: why a new approach is needed. Am Heart J 2010;159:245-57.

20. Galiè N, Olschewski H, Oudiz RJ, et al. Ambrisentan for the treatment of pulmonary arterial hypertension: results of the ambrisentan in pulmonary arterial hypertension, randomized, double-blind, placebo-controlled, multicenter, efficacy (ARIES) study 1 and 2. Circulation 2008;117:3010-9.

21. Eid H, de Bold ML, Chen JH, de Bold AJ. Epicardial mesothelial cells synthesize and release endothelin. J Cardiovasc Pharmacol 1994;24:715-20.

22. Kohan DE. Endothelins in the normal and diseased kidney. Am J Kidney Dis 1997;29:2-26.

23. Ehrenreich H, Anderson RW, Fox CH, et al. Endothelins, peptides with potent vasoactive properties, are produced by human macrophages. J Exp Med 1990;172:1741-8.

24. Drakopoulos A, Goumenos DS, Vlachojannis JG, Tsakas S. Endothelin receptors in the kidney of patients with proteinuric and non-proteinuric nephropathies. Ren Fail 2006;28:461-7.

25. Dufek B, Meehan DT, Delimont D, et al. Endothelin A receptor activation on mesangial cells initiates Alport glomerular disease. Kidney Int 2016;90:300-10.

26. Barton M. Reversal of proteinuric renal disease and the emerging role of endothelin. Nat Clin Pract Nephrol 2008;4:490-501.

27. Barton M. Therapeutic potential of endothelin receptor antagonists for chronic proteinuric renal disease in humans. Biochim Biophys Acta 2010;1802:1203-3.

28. Ohta K, Hirata Y, Shichiri M, et al. Urinary excretion of endothelin-1 in normal subjects and patients with renal disease. Kidney Int 1991;39: $307-11$.

29. Tan RJ, Zhou L, Zhou D, Lin L, Liu Y. Endothelin receptor a blockade is an ineffective treatment for adriamycin nephropathy. PLoS ONE 2013;8: e79963.

30. Ikebe M, Nonoguchi H, Nakayama Y, et al. Regulation of endothelinconverting enzyme 1 in nephrotic syndrome in rats. Nephron Exp Nephrol 2003;94:e137-45.

31. Mundel P, Reiser J. Proteinuria: an enzymatic disease of the podocyte? Kidney Int 2010;77:571-80.

32. Shimada M, Ishimoto T, Lee PY, et al. Toll-like receptor 3 ligands induce CD80 expression in human podocytes via an NF-kB-dependent pathway. Nephrol Dial Transplant 2012;27:81-9.

33. Henry J, Miller MM, Pontarotti P. Structure and evolution of the extended B7 family. Immunol Today 1999;20:285-8. 


\section{Articles | Kino et al.}

34. Chambers CA, Allison JP. Costimulatory regulation of T cell function. Curr Opin Cell Biol 1999;11:203-10.

35. Yu CC, Fornoni A, Weins A, et al. Abatacept in B7-1-positive proteinuric kidney disease. N Engl J Med 2013;369:2416-3.

36. Mishra OP, Kumar R, Narayan G, et al. Toll-like receptor 3 (TLR-3), TLR-4 and CD80 expression in peripheral blood mononuclear cells and urinary CD80 levels in children with idiopathic nephrotic syndrome. Pediatr Nephrol 2017;32:1355-61.
37. Shimo T, Adachi Y, Yamanouchi S, et al. A novel nuclear factor $\kappa B$ inhibitor, dehydroxymethylepoxyquinomicin, ameliorates puromycin aminonucleosideinduced nephrosis in mice. Am J Nephrol 2013;37:302-9.

38. Mundel P. Podocyte-targeted treatment for proteinuric kidney disease. Semin Nephrol 2016;36:459-62.

39. Garsen M, Lenoir O, Rops AL, et al. Endothelin-1 induces proteinuria by Heparanase-mediated disruption of the glomerular Glycocalyx. J Am Soc Nephrol 2016;27:3545-51. 